\title{
Cenglestato
}

\section{Crescimento, diversidade e sobrevivência: o conceito de vitalidade aplicado em um estudo cientométrico}

\author{
Maria de Fátima Santos Maia \\ Doutora; Universidade Federal do Rio Grande (FURG), Rio Grande, RS, Brasil; \\ fatima-maia@uol.com.br \\ Sônia Elisa Caregnato \\ Doutora; Universidade Federal do Rio Grande do Sul (UFRGS), Porto Alegre, RS, Brasil; \\ sonia.caregnato@ufrgs.br
}

\begin{abstract}
Resumo: Aborda a estrutura e a dinâmica das atividades de produção científica na área das Ciências da Saúde no Brasil. Partiu-se do pressuposto de que a vitalidade da ciência pode ser identificada através de características sobre produtividade de autores, longevidade de instituições, diversidade temática e canais de divulgação. Por meio da identificação dessas características específicas, foram apontados indícios que representam diferentes graus de vitalidade. Analisando 117.521 artigos de autores brasileiros publicados entre 1987 e 2011 em periódicos indexados pela base de dados bibliográfica Medline, foram identificadas características de vitalidade em cada um dos 27 estados da federação. Os resultados revelaram que, na área das Ciências da Saúde no Brasil, há muita disparidade entre os estados e que os maiores graus de vitalidade científica estão localizados nos estados de São Paulo, Rio de Janeiro e Rio Grande do Sul.
\end{abstract}

Palavras-chave: Comunicação científica. Padrões de comunicação científica Brasil. Ciências da Saúde. Cientometria.

\section{Introdução}

Nas últimas décadas, o Brasil tem construído uma ampla e reconhecida base científica, impulsionada, principalmente, por políticas de incentivo financeiro e programas para formação de pesquisadores (CRUZ, 2012). As decisões de como implantar estas políticas e programas, assim como o encaminhamento das suas melhorias e ajustes se apoiam, entre outros parâmetros, nas métricas sobre o desempenho de pesquisadores individuais, departamentos acadêmicos e/ou instituições. Por outro lado, a comunidade científica se declara insatisfeita com a construção de medidas que resultam na determinação de rótulos do tipo "os mais produtivos" ou "os de maior impacto" (LANE, 2010). Entre a insatisfação da 
comunidade científica e a necessidade de gerar informações confiáveis, encontram-se os estudos cientométricos que, a partir da interpretação de diferentes modelos e medidas, buscam revelar as características das unidades e processos envolvidos. Os modelos estruturais evidenciam regularidades na literatura científica através das características, por exemplo, dos autores e suas instituições de vínculo, dos termos que representam o conteúdo, dos periódicos utilizados como canais de comunicação ou das citações recebidas em trabalhos posteriores. Os modelos dinâmicos investigam as alterações ocorridas em um período de tempo, como surgimento de um campo, padrões de produtividade de um país ou região, fusão ou cisão de equipes (BÖRNER et al., 2012).

Atualmente, impulsionado pelo amadurecimento da área e desenvolvimento de métodos e ferramentas específicas, identifica-se um aumento no interesse por modelos que contemplam análises mistas, isto é, que abordam a estrutura e também a dinâmica das atividades científicas (GREENBERG, 2009; DING; CRONIN, 2011; MALIet al., 2012; WAINER; VIEIRA, 2013).

Neste trabalho, além da descrição da estrutura da produção e identificação de alterações e recorrências no período entre 1987 até 2011 (dinâmica), também se optou por buscar parâmetros que indicassem características de vitalidade nas atividades de produção científica em ciências da saúde de instituições localizadas nos 27 estados do Brasil. Tais características foram observadas em uma série de desdobramentos decorrentes da produtividade de pesquisadores e instituições, da diversidade temática abordada nas publicações, da quantidade de cientistas atuantes e da longevidade das instituições.

O conceito de vitalidade não tem sido abordado em estudos sobre a produção científica brasileira. Portanto, considerou-se adequado explorar esta lacuna através de um estudo cientométrico, considerando observações e análises sobre a estrutura e dinâmica de unidades que compõem a literatura publicada nas ciências da saúde, ou seja, das características da comunicação científica da área. Em síntese, partindo do pressuposto que a vitalidade se evidencia através dos fenômenos de crescimento, sobrevivência e diversidade, a proposta desta pesquisa foi identificar estas características na produção científica, em ciências da saúde, dos 27 estados do Brasil. 


\section{0 conceito de vitalidade em estudos cientométricos}

A vitalidade é um termo empregado em diferentes campos de estudos, entretanto, mesmo em contextos distintos, está sempre associada à ideia de energia, vigor, capacidade de viver, crescer e se multiplicar. Na biologia, talvez a área na qual apareça com mais frequência, vitalidade é usada como medida de análise do desenvolvimento de diferentes espécies, sejam microrganismos (PARDO; GALVAGNO; CERRUTTI, 2009) ou recifes de corais (KIKUCHI; LEAO; OLIVEIRA, 2010). A medicina utiliza um instrumento padronizado para avaliar a percepção de saúde de indivíduos, denominado Índice de Energia e Vitalidade (BRAZIER et al., 1992). A mesma concepção pode ser encontrada em estudos sobre satisfação profissional de professores, segundo os quais, a partir de indicadores como produtividade e envolvimento no trabalho, foi elaborado um Índice de Vitalidade Docente (DANKOSKI et al., 2011).

Em trabalhos sobre atividades relacionadas à produção científica, foco de interesse desta pesquisa, a ideia de vitalidade tem sido usada, principalmente, a partir do cálculo da idade média de citações. Partindo do pressuposto que citar trabalhos recentes significa incorporação rápida de novas ideias, velocidade em alcançar consenso paradigmático, indicando, portanto, vitalidade nas atividades desenvolvidas pela comunidade de um determinado campo do conhecimento. Assim, estes estudos consideram que quanto mais novas as referências citadas em determinado campo do conhecimento, mais rápido os pesquisadores alcançam um consenso paradigmático e esta característica estaria relacionada com o nível de vitalidade do campo (KLAVANS; BOYACK, 2008; SANDSTRÖM; SANDSTRÖM, 2009; BOYACK; KLAVANS, 2010). É possível dizer que a ideia de vitalidade, analisada através das citações, se apoia na etimologia dos adjetivos latinos citus, cita, citum que indicam sacudir, provocar, colocar em movimento e estar vivo, pois a vitalidade de um campo estaria na capacidade de publicar trabalhos que rapidamente gerem novas citações no campo (CASTIEL; SANZ-VALERO, 2007). 
Em um estudo sobre a ciência produzida nos Estados Unidos, a diversidade temática e variedade de paradigmas foram características associadas com a ideia de vitalidade (COMMITTEE ON ASSESSING BEHAVIORAL, 2006). A intensa discussão dentro de um campo, a partir de diferentes paradigmas, estaria relacionada com a sua vitalidade. Entretanto, não se pode deixar de mencionar aqui a existência de peculiaridades das disciplinas, pois nas ciências econômicas, por exemplo, o consenso paradigmático é um objetivo perseguido, da mesma forma que a ausência de um paradigma dominante estaria relacionado com o enfraquecimento do campo (COLE; COLE; SIMON, 1981; COLE, 1983; COMMITTEE ON ASSESSING BEHAVIORAL, 2006).

Ainda no contexto dos estudos cientométricos que abordaram o conceito de vitalidade a partir das citações, é possível referir o indicador Impact Vitality (RONS; AMEZ, 2009). Esta métrica pressupõe que o aumento do número das citações para um pesquisador, tema, instituição ou país indica seu grau de vitalidade. Através da aplicação de uma fórmula específica para analisar as citações recebidas durante um período, o indicador Impact Vitality pode resultar em valores ascendentes que demonstram grau de vitalidade maior do que quando permaneceram com valor igual ou menor. Entretanto, assim como é importante estar atento às especificidades das disciplinas, é preciso também considerar a existência de publicações e autores que representam marcos fundadores em campos específicos e, portanto, são citados por longos períodos, como Charles Darwin na biologia, ou Alfred Lotka na ciência da informação.

Em outro trabalho relacionado ao tema vitalidade, físicos do Laboratório Nacional de Los Alamos (Novo México, EUA) construíram um modelo para analisar o surgimento e desenvolvimento de seis diferentes campos de estudos nas áreas da Física, Biomedicina, Computação e Nanotecnologia. Através de campos de estudos bem específicos (inflação cósmica, cordas cósmicas, doenças priônicas, gripe H5N1, nanotubos de carbono e computação quântica), os autores afirmam que a vitalidade de um campo está associada ao aumento da produtividade e número de autores vinculados. $\mathrm{O}$ aumento da produtividade foi considerado como indicador da existência de temas potencialmente abertos para serem explorados. Na visão destes autores, a existência de potencialidades propor- 
ciona que novos pesquisadores sejam incorporados pelo campo, indicando a existência de oportunidades para novas investigações. Da mesma forma, os fluxos de retrocesso na produtividade e a redução do número de autores vinculados são indicativos de saturação e esgotamento de oportunidades de pesquisas e, portanto, opondo-se à ideia de vitalidade (BETTENCOURT et al., 2008).

Maior produtividade e número de autores em campos científicos também foram mencionados como características de vitalidade no relatório da Academia Nacional de Ciências dos Estados Unidos (COMMITTEE ON ASSESSING BEHAVIORAL, 2006). Os pesquisadores consideraram que a vitalidade de um campo científico poderia ser apreendida a partir da identificação das seguintes características: cientistas estabelecidos que começam a desenvolver trabalhos em um diferente campo de estudos; aumento da quantidade de matrículas de alunos interessados em novos cursos e programas; pesquisadores iniciantes que são reconhecidos por terem carreiras promissoras e que optam por buscar novos conceitos, métodos ou linhas de investigação; aumento do número de publicações em um campo; aumento de citações para publicações de um determinado campo de estudos; artigos sobre um novo campo que começam a aparecer em revistas importantes; criação de novos periódicos; ideias que passam a ser adotadas por diferentes campos; pesquisadores de diferentes áreas que começam a colaborar em trabalhos que tratam de problemas semelhantes (COMMITTEE ON ASSESSING BEHAVIORAL, 2006).

Assim, a partir das reflexões sobre estudos anteriores que já abordaram o conceito de vitalidade aplicado ao entendimento dos processos de produção científica, somado ao significado intrínseco do termo, optou-se neste trabalho em desenvolver uma análise que contemplasse três dimensões: crescimento, diversidade e sobrevivência da literatura de uma área. A ideia de crescimento foi associada com a produtividade científica de autores e instituições, a diversidade foi relacionada aos diferentes periódicos e temas dos artigos, e a sobrevivência com a longevidade de instituições e publicações em periódicos de alto prestígio. Reunidos, estes parâmetros permitiram traçar um perfil de vitalidade para as atividades científicas de cada estado do Brasil. 
No que se refere a produtividade científica, é possível afirmar que se trata do mais simples entre os indicadores utilizados em estudos cientométricos, podendo ser associado ao grau de dinamismo das atividades de pesquisadores, campos de estudos, países ou instituições (CALLON; COURTIAL; PENAN, 1995). O crescimento do número de publicações é resultado de processos sucessivos de acumulação, que apontam para a intensidade do esforço despendido por, entre outros, pesquisadores ou instituições. Nos estudos cientométricos, estes processos de acumulação também são interpretados como "capital intelectual", composto por capital humano, estrutural e relacional (BUCHELI et al., 2012). O nível de capital acaba se refletindo em vantagens competitivas, pois a capacidade de produção no momento presente aumenta as chances de alcançar, no futuro, no mínimo o mesmo patamar, formando assim um ciclo de acumulação de capital intelectual (BUCHELI et al., 2012). Autores com maior capital intelectual têm mais chances de publicar e, consequentemente, ser mais citados e visíveis dentro do seu campo de estudos (URBIZAGÁSTEGUI ALVARADO, 2009). Neste sentido, acredita-se que uma instituição que apresenta uma taxa de produtividade maior tem maior grau de vitalidade. A produtividade científica também reflete crescimento, permitindo alcançar maiores chances de sobrevivência. Além disso, produtividade e crescimento também indicam movimento que, consequentemente, revela a existência de energia e vitalidade. No âmbito biológico, dizemos que alguma matéria está viva quando ela continua a movimentar-se, realizando trocas, de qualquer natureza, com seu meio ambiente por um longo período, isto é, trata-se do oposto de uma matéria inanimada e imutável. "O estado estável de um organismo vivo é estar morto." (SCHRÖDINGER apud GLEICK, 2013, p. 291). Assim, partindo da importância de analisar características de movimento e crescimento, a produtividade foi considerada o primeiro parâmetro para a determinação de maior ou menor grau da vitalidade de instituições e estados.

Diversidade constitui a segunda dimensão escolhida para analisar a vitalidade da produção científica brasileira no contexto desta investigação. Nas ciências biológicas é reconhecida a correlação entre diversidade e viabilidade de sobrevivência (JABLONSKI, 2005; FOOTE et al., 2008). Neste trabalho, a di- 
versidade de autores e a diversidade de instituições têm relação com os parâmetros utilizados no estudo anteriormente citado, sobre a produção científica na área da Física, na qual a vitalidade de um campo ou de uma instituição foi associada não só ao aumento da produtividade, mas também ao número de autores vinculados. Novos pesquisadores se incorporam a instituições e temas com potencial para serem explorados (BETTENCOURT et al., 2008). O compartilhamento de tarefas, informações e competências é um fator relevante para o sucesso de pesquisas acadêmicas. A colaboração entre pesquisadores pode proporcionar economia de tempo, recursos financeiros e materiais. Além disso, hoje em dia, há consenso sobre a importância das colaborações entre pesquisadores para o avanço da ciência, pois a justaposição de diferentes ideias tem potencial significativo para a promoção de pesquisas com maior grau de inovação e impacto social (MALI et al., 2012). Sendo assim, a quantidade de autores produtivos vinculados com as instituições de cada estado pode contribuir nas análises de vitalidade. A diversidade de instituições segue a mesma lógica dos autores e temas, isto é, se um estado mostra um fluxo de aumento de diferentes instituições que se incorporam ao cenário das suas atividades de pesquisa, significa a existência de potencialidades e capacidade física e intelectual para incorporação também de novos temas, indicando maior grau de interdisciplinaridade (IRIBARREN-MAESTRO, 2006). Acrescenta-se ainda que disciplinas científicas representam a estrutura organizacional e cognitiva onde acontece a produção do conhecimento (MALI et al., 2012). Além disso, cada área do conhecimento tem sua própria terminologia para definir conceitos e explicar os processos e fenômenos de que se ocupam (MAYR, 2008). O uso da diversidade de temas se apoia no pressuposto de que uma instituição deve e necessita estar aberta para acolher diferentes abordagens de pesquisa. As instituições permitem, simultaneamente, transmitir a ciência que está sendo produzida e acolher a que ainda não foi feita (PORTOCARRERO, 2009). Então, partindo do pressuposto de que a variedade temática é uma característica importante na construção do conhecimento, considera-se pertinente o seu uso para compor as análises de vitalidade. Assim, a diversidade de autores, de temas e instituições foi associada à ideia de vitalidade. 
O terceiro parâmetro utilizado para compor as análises de vitalidade foi a longevidade. Neste caso, o tempo de permanência das atividades de produção foi o referencial adotado, partindo do pressuposto que fazer ciência não é um processo fácil e tampouco rápido. Em outras palavras, uma instituição que consegue permanecer ativa durante muito tempo indica a existência de condições humanas e materiais que permitem sua permanência no cenário produtivo. Portanto, os estados que reúnem mais instituições ativas por um longo período foram considerados com maior grau de longevidade.

\section{Procedimentos metodológicos}

Os dados analisados foram coletados na base bibliográfica Medline, que tem qualidade e prestígio reconhecidos junto à comunidade científica da área das ciências da saúde (SANTOS, 2010; COBO et al., 2011). Aceita como a mais importante fonte de informações bibliográficas da área médica e biomédica, a Medline é um subgrupo da base bibliográfica PubMed, sendo mantida e disponibilizada pela National Library of Medicine (NLM), que é apontada como a maior agência financiadora de pesquisas do mundo (LEYDESDORFF; ROTOLO; RAFOLS, 2012). O conteúdo da base é atualizado permanentemente, sendo que em 2013, 51 periódicos brasileiros eram indexados pela Medline.

A estratégia empregada para reunir os artigos publicados por pesquisadores brasileiros consistiu em buscar pelas palavras "Brasil" ou "Brazil" no campo affiliation, que mostra informações sobre o endereço de vínculo e/ou contato do primeiro autor. Como este campo só foi incorporado na Medline em 1987, decidiu-se limitar o período da pesquisa a partir deste ano. Esta opção também se apoia no fato de que a década de 80, no Brasil, foi o marco final do regime militar e ponto de partida para um novo capítulo da história do país. A partir de 1985, no período denominado de Nova República, o Brasil inaugurou uma fase de mudanças em todos os setores, incluindo também a maneira de fazer ciência e pesquisa. Assim, partindo destes pressupostos, optou-se por limitar o escopo temporal para coleta de dados em 25 anos $(1987-2011)$. 
Após os procedimentos de coleta, todas as referências bibliográficas compuseram um banco de dados que foi organizado no software EndNote (versão X7). O uso desta ferramenta, além de permitir a importação das referências, ofereceu também recursos que facilitaram o processo de limpeza dos dados, tais como a padronização da grafia dos nomes das instituições, o agrupamento de trabalhos com características em comum e a exportação dos registros para planilhas do software Microsoft Excel. O Tableau foi utilizado na criação das figuras deste trabalho.

No que se refere às unidades de análise, decidiu-se utilizar as instituições, autores, artigos, títulos dos periódicos e o escopo temático dos periódicos. A escolha da primeira unidade (instituições) está apoiada no caráter social da ciência. As instituições ocupam posição destacada no apoio e financiamento das atividades relacionadas à produção de conhecimento e permitem, simultaneamente, transmitir a ciência que está sendo produzida e acolher a que ainda não foi feita (PORTOCARRERO, 2009). É através delas que são organizados eventos, publicados periódicos, aprovadas resoluções, concedidos prêmios e discutidos problemas específicos de interesse da comunidade que representam (ZIMAN, 1979). Além disso, as características das instituições científicas podem revelar aspectos específicos da cultura, da época, da história, do meio e do país, ou seja, o contexto no qual o conhecimento é ou foi construído (PORTOCARRERO, 2009). Sendo assim, é possível afirmar que a vitalidade da ciência pode estar relacionada com alterações que ocorrem na estrutura e na dinâmica das relações entre as instituições científicas.

A utilização da segunda unidade de análise (autores) está fundamentada na ideia de que quando um paradigma científico se estabelece, um número significativo de pesquisadores, convencidos da fecundidade da nova concepção, se interessa pelo novo modelo de praticar a ciência (KUHN, 2005). Além disso, os campos de investigação que agregam um número expressivo de cientistas ativos têm mais chances de conquistar maiores avanços e atrair novos e qualificados pesquisadores (COMMITTEE ON ASSESSING BEHAVIORAL, 2006). Portanto, é possível inferir que a quantidade de autores vinculados a um campo de estudos ou uma instituição está relacionada com a sua vitalidade. 
A opção pela terceira unidade (artigos) está relacionada com a segunda (autores), pois quando uma linha de pesquisa se desenvolve demonstrando vigor e energia, mais trabalhos são produzidos. Estudos anteriores mostraram existir uma correlação positiva entre a taxa de crescimento de autores e artigos, ou seja, um vínculo entre o número de pesquisadores e aumento da produtividade (GLÄNZEL; LETA; THUS, 2006; BETTENCOURT et al., 2008).

A aplicação da quarta unidade de análise (títulos dos periódicos) se deve ao pressuposto de que cada área do conhecimento tem seus próprios canais de comunicação e os periódicos utilizados por determinadas comunidades científicas podem revelar características da estrutura cognitiva da área, assim como o nível de inserção internacional e visibilidade (TENOPIR; KING, 2000; POBLACIÓN et al., 2011). A identificação da cobertura temática dos títulos dos periódicos permite a identificação de áreas e campos de estudo. Além disso, a quantidade de artigos publicados em periódicos internacionais pode indicar um nível de inserção internacional (LEITE; MUGNAINI; LETA, 2011).

Portanto, partindo da ideia de que a ciência é construída por indivíduos institucionalmente vinculados, que se revela nos produtos gerados das suas atividades e adquire sentido através dos canais e temas utilizados, é válido analisála a partir destes sujeitos (autores), dos seus vínculos sociais (instituições), dos seus produtos (artigos), e dos canais e temas de pesquisa (periódicos).

Para operacionalizar o grau de vitalidade de cada estado da federação e obtenção de valores que os representassem, foi necessário estabelecer parâmetros específicos, sendo que as dinâmicas das alterações ocorridas ao longo do tempo foram analisadas através da taxa de crescimento geral de artigos publicados, da quantidade de autores vinculados com as instituições de cada estado e da diversidade temática, isto é, as diferentes classificações temáticas dos periódicos utilizados nas publicações.

As análises sobre a quantidade de instituições longevas abrangeram o nível de estrutura e dinâmica, pois foi necessária uma análise longitudinal (25 anos) para depois contabilizar a quantidade de instituições que permaneceram ativas em cada estado, por todo o período. 
As características de estrutura focaram as quantidades absolutas de instituições produtivas, número de instituições do estado que registraram autores prolíficos e que publicaram em periódicos de alto Fator de Impacto (FI).

Foram consideradas produtivas aquelas instituições que alcançaram média total igual ou superior a 250 artigos publicados. Como o período de cobertura dos dados cobre o intervalo de 25 anos, acredita-se que a média de 10 artigos/ano é um parâmetro mínimo para ser considerado como produtividade institucional. Considerou-se como prolíficos aqueles autores que publicaram 200 artigos ou mais no período analisado. A opção por utilizar esta quantidade apoiou-se na revisão de literatura, que revelou dois artigos que avaliaram a produção científica de autores da área da saúde em extensos períodos, sendo que ambos apontam que os mais produtivos totalizaram 219 publicações (OLIVEIRA et al., 2011; BOUCHARD et al., 2015). Para classificar os periódicos conforme o Fator de Impacto, foi consultado o Journal Citation Reports de 2011. Entende-se que publicar em periódicos de alto impacto amplia a inserção de pesquisas e pesquisadores em diferentes comunidades acadêmicas.

O Quadro 1, abaixo, apresenta as descrições de cada parâmetro, sendo que se optou em designar um código para cada um, utilizando a letra V (Vitalidade) e um número sequencial (V1, V2, V3...V7). É possível dizer que todos estes parâmetros se correlacionam com as características de vitalidade, sendo que V1, V5 e V6 conotam o crescimento, V2, V3, V7 diversidade e V4 longevidade. Os parâmetros V1, V2 e V3 foram obtidos através de análises sobre a dinâmica da produção científica e os resultados mostram taxas de crescimento com medidas que variam entre valores negativos até 60. Os parâmetros V4, V5, V6 e V7 referem-se as análises de estrutura sendo que os resultados são apresentados em valores absolutos que variam entre zero e dez. 
Quadro 1 - Descrição dos códigos e parâmetros utilizados para analisar as características de vitalidade da produção científica dos estados do Brasil (1987-2011)
Código
Descrição dos parâmetros

Média da taxa geométrica de crescimento anual do número de artigos publicados por estado. A fórmula usada nos cálculos da taxa de crescimento é a mesma utilizada pelo IBGE, para análises do crescimento populacional no Brasil:

V1

$$
\sqrt[n]{\frac{P(t+n)}{P(t)}} \times 100
$$

$n=$ número de anos do período.

$P(t)=$ número de inicial de artigos, instituições ou autores.

$P(t+n)=$ número final de artigos, instituições ou autores.

V2 Média da taxa geométrica de crescimento anual do número de autores. A fórmula usada nos cálculos foi a mesma do parâmetro V1.

V3 Média da taxa geométrica de crescimento temático anual. A fórmula usada nos cálculos foi a mesma do parâmetro V1.

V4 Número de instituições longevas que indica a quantidade de instituições que publicaram em todo o período de abrangência deste estudo, ou seja, pelo menos um artigo por ano no período entre 1987-2011.

V5

Número de instituições produtivas (número de artigos $\geq 250$ ).

V6 Número de instituições do estado que registraram autores prolíficos (número de artigos publicados $\geq 200$.

V7 Quantidade de instituições do estado cujos autores publicaram em periódicos com alto Fator de Impacto (FI).

Fonte: Dados da pesquisa

Por meio da identificação das características específicas relativas a crescimento, diversidade e longevidade, e sua operacionalização enquanto conjunto de sete parâmetros, buscou-se aferir o grau de vitalidade da produção científica de cada estado do Brasil. Os achados são relatados a seguir. 


\section{Resultados}

As primeiras análises revelaram que em 25 anos, os autores que informaram vínculo com instituições brasileiras publicaram 117.521 artigos em periódicos indexados na Medline. Essa quantidade corresponde a 0,92\% de todo o conteúdo da base no mesmo período (1987-2011). Estes artigos foram publicados por 155.248 autores vinculados com 481 instituições, em 3.950 diferentes periódicos que abrangem 1.583 subáreas das ciências da saúde (MAIA, 2014).

A Figura 1 representa o grau de vitalidade para cada estado brasileiro. Nela é possível observar que o Amazonas e Paraná alcançaram maiores escores. Entretanto, conforme os parâmetros desta pesquisa, para ser considerados com vitalidade plena os estados precisariam apresentar valores diferentes de zero em todas as medidas $(\mathrm{V} 1, \mathrm{~V} 2, \mathrm{~V} 3 \ldots \mathrm{V} 7 \neq 0)$ e somente quatro apresentaram esta característica. Sendo assim, é possível afirmar que apenas nas instituições de São Paulo (SP), Rio de Janeiro (RJ), Rio Grande do Sul (RS) e Pernambuco (PE), no período analisado, houve vitalidade plena nas atividades de produção científica em ciências da saúde. Estes quatro estados, mesmo não apresentando os maiores valores na maioria dos parâmetros, tiveram uma distribuição mais equilibrada no conjunto das cores que representam crescimento, diversidade e sobrevivência e, consequentemente, vitalidade. 
Figura 1 - Características de vitalidade da produção científica em ciências da saúde nos 27 estados do Brasil, 1987-2011 ( $\mathrm{n}=117.521)$

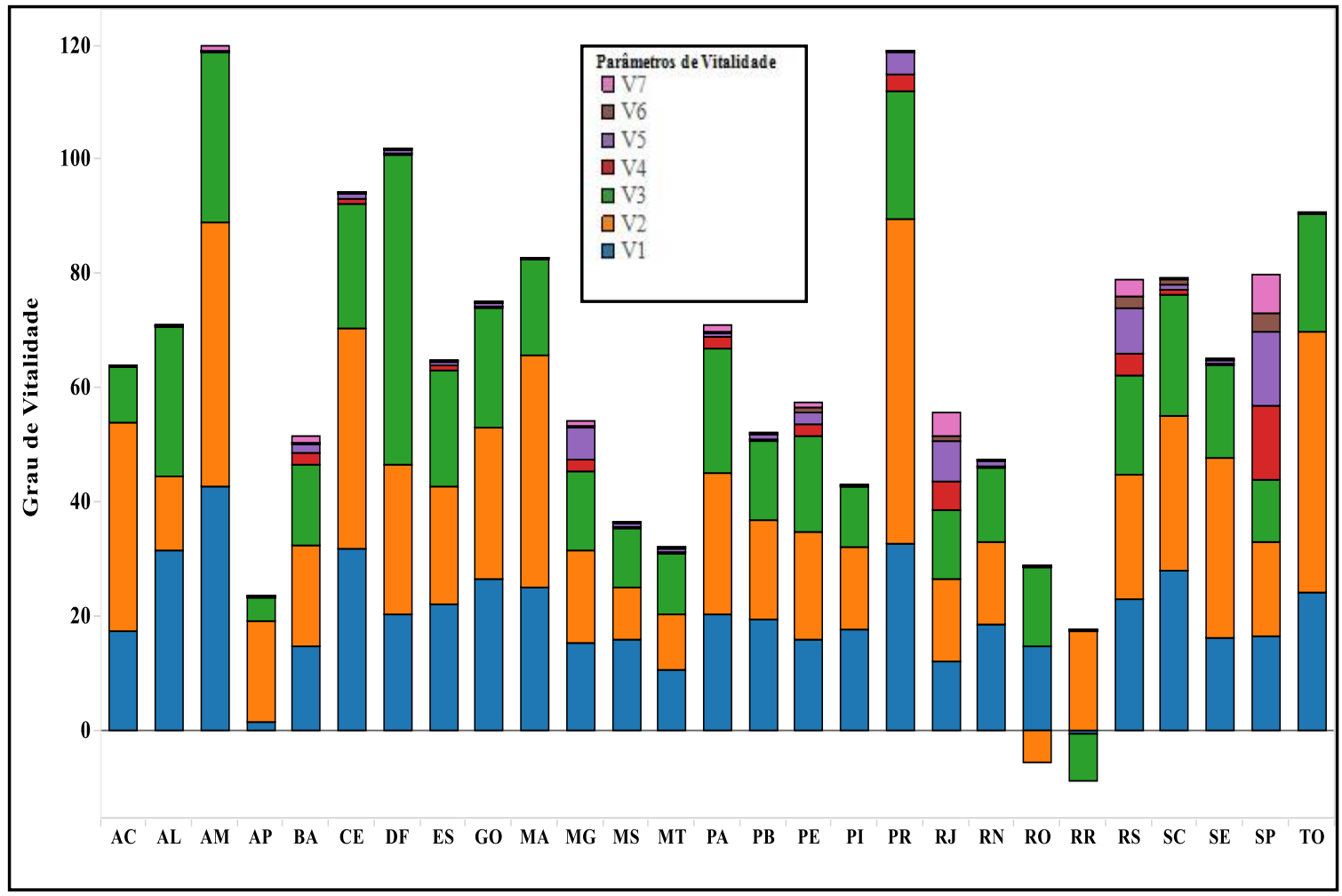

Fonte: Dados da pesquisa

Legenda: V1=média da taxa geométrica de crescimento anual do número de artigos publicados por estado; V2=média da taxa geométrica de crescimento anual do número de autores; V3=média da taxa geométrica anual de crescimento temático; V4=número de instituições longevas; V5=número de instituições produtivas; V6=número de instituições do estado que registraram autores prolíficos; $\mathbf{V 7 = q u a n t i d a d e ~ d e ~ i n s t i t u i c ̧ o ̃ e s ~ c u j o s ~ a u t o r e s ~ p u b l i c a r a m ~ e m ~ p e r i o ́ d i c o s ~}$ com alto FI.

A Figura 1 mostra, ainda, que Roraima registrou valores negativos nas taxas de crescimento de artigos publicados e diversidade temática; Rondônia apresentou média inferior a zero na taxa de crescimento de autores; Amapá, Mato Grosso e Mato Grosso do Sul não registraram valores negativos, mas os resultados alcançados pelos pesquisadores e instituições destes estados demonstram um grau reduzido nos parâmetros de crescimento, diversidade e sobrevivência. Este resultado aponta a ausência de vitalidade na produção científica em ciências da saúde das instituições destes estados.

A Figura 2 mostra, separadamente, o resultado de cada parâmetro para todos os estados. É possível verificar, através da quantidade de barras horizontais, que autores prolíficos (V6) e publicações em periódicos de alto Fator de 
Impacto (V7) foram os parâmetros mais difíceis de serem alcançados pelas instituições da maioria das 27 unidades da federação.

Figura 2 - Grau de vitalidade conforme cada parâmetro (n=117.521 artigos)

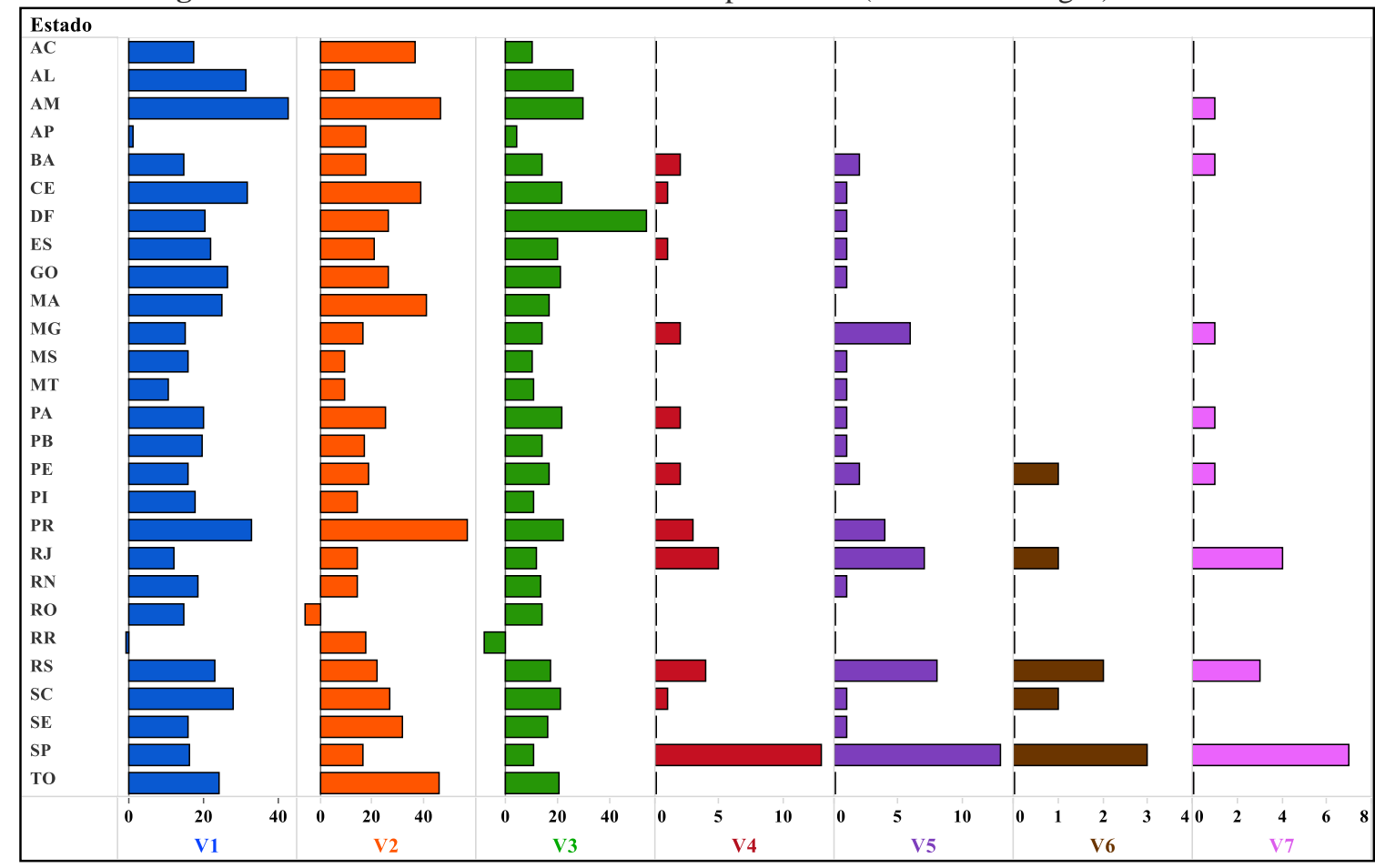

Fonte: Dados da pesquisa

No que se refere à média geométrica de crescimento anual do número de artigos publicados (V1), a maioria dos estados da federação registrou atividades positivas, exceto Roraima, que apresentou escore negativo. O Amapá teve um crescimento pouco significativo, apresentando valor pouco acima de um $(1,39)$. O Amazonas se destacou em relação aos demais, mostrando um escore acima de 40. Este resultado permite dizer que, na área das Ciências da Saúde e no período analisado, os pesquisadores vinculados com instituições do Amazonas se mantiveram bastante ativos, publicando um volume crescente de artigos em periódicos indexados pela base de dados Medline.

No que se refere à taxa de crescimento do número de autores (V2) se destacaram os estados do Paraná e Amazonas, enquanto Rondônia teve uma média negativa neste mesmo parâmetro. Embora tenha se observado que as taxas de crescimento de autores vinculados com instituições do Tocantins e Maranhão também tenham sido altas, ao conferir os escores absolutos verificou-se 
que estes estados apresentaram pouca atividade antes de 2000, resultando em uma média final fortemente influenciada pelos valores dos últimos anos.

Em relação a diversidade temática (V3), o Distrito Federal apresentou a maior taxa. Esse resultado indica que, dentro da grande área das Ciências da Saúde, os pesquisadores do Distrito Federal atuam com maior amplitude de subtemas. Conforme as análises mais específicas, a região Centro-Oeste se destacou na produção científica que contempla principalmente os subtemas saúde pública e parasitologia (MAIA, 2014).

Nas análises sobre as instituições que permaneceram ativas em todo o período (V4), 11 estados registraram valor diferente de zero. O destaque maior foi para São Paulo, com 13 instituições ativas, seguido pelo Rio de Janeiro $(n=5)$ e Rio Grande do Sul $(n=4)$. Este resultado permite dizer que a ciência produzida nestes estados está mais madura, consequência de instituições com estruturas mais consolidadas, o que permitiu que as atividades de pesquisa encontrassem espaço e condições adequadas para seu desenvolvimento.

Sobre as instituições produtivas (V5), verificou-se que os estados de São Paulo ( $n=13)$, Rio Grande do Sul ( $n=8)$, Rio de Janeiro (n=7), Minas Gerais $(n=6)$ e Paraná $(n=4)$ apresentaram maior quantidade de instituições que publicaram um total igual ou superior a 250 artigos no período (média 10/ano). Comparando com as instituições longevas, São Paulo apresentou a mesma quantidade para os dois parâmetros (V4 e V5 = 13), entretanto, nos estados do Rio de Janeiro, Rio Grande do Sul, Minas Gerais e Paraná o número de instituições produtivas foi superior às longevas.

Quanto ao parâmetro V6, que se refere ao número de instituições cujos autores publicaram mais de 200 artigos no período (prolíficos), se destacaram, no Rio Grande do Sul, a Universidade Federal do Rio Grande do Sul (UFRGS) e Universidade Federal de Pelotas (UFPel), a Fiocruz de Pernambuco (Centro de Pesquisas Aggeu Magalhães), a Fiocruz do Rio de Janeiro, a Universidade Federal de Santa Catarina (UFSC) e, em São Paulo, a Universidade Federal de São Paulo (UNIFESP), Universidade de São Paulo (USP) e Hospital A.C. Camargo. Em relação aos demais parâmetros, este foi o que totalizou maior quantidade de zeros, em 22 dos 27 estados os pesquisadores das ciências da saúde não se ca- 
racterizaram como muito produtivos de acordo com os parâmetros utilizados nesta investigação.

Em referência ao último parâmetro (V7), destacaram-se os pesquisadores vinculados com as instituições de oito estados: Amazonas, Bahia, Minas Gerais, Pará, Pernambuco, Rio de janeiro, Rio Grande do Sul e São Paulo. Estes pesquisadores publicaram artigos em periódicos de maior fator de impacto, resultado que pode ser interpretado como um maior grau de inserção internacional.

Assim, os resultados revelam que há muita disparidade entre os estados brasileiros no que se refere ao crescimento anual do número de artigos, autores e temas, à produtividade e longevidade de instituições e ao número de instituições que registraram autores prolíficos e que publicam em revistas de alto fator de impacto na área das Ciências da Saúde. Além disso, observou-se que os maiores graus de vitalidade científica estão localizados nos estados de São Paulo, Rio de Janeiro e Rio Grande do Sul.

\section{Conclusões}

De maneira geral a produção científica brasileira, medida através dos artigos publicados em periódicos indexados na Medline entre 1987 e 2011, cresceu. Este resultado aponta um esforço de todos os envolvidos no processo de produção científica em Ciências da Saúde no Brasil.

Conforme já mencionado, em estudos cientométricos que abordaram o conceito de vitalidade, foi referido que o aumento de produtividade e número de pesquisadores pode ser interpretado como indicador da existência de temas e espaços de pesquisa potencialmente abertos e promissores (BETTENCOURT et al., 2008).

Se por um lado observou-se um forte crescimento das atividades de pesquisa em geral, nos estados do Amazonas e Paraná, por outro lado, os resultados obtidos pelas instituições e pesquisadores de Roraima, Rondônia e Amapá indicam a necessidade de rever os processos de produção científica na área da saúde nestes estados. É preciso reconhecer a urgência de encaminhar políticas de in- 
centivo financeiro, de estrutura física, de tecnologia e programas de formação de pesquisadores nestes estados. Estas ações poderão reduzir as desigualdades existentes entre estes três estados e os demais, proporcionando maior grau de vitalidade na produção de conhecimento no Brasil como um todo.

Trabalhos futuros que contemplem os mesmos parâmetros e o universo desta pesquisa poderão revelar se os estados nos quais se verificou o crescimento de produção e pesquisadores alcançarão os escores considerados com indicadores de vitalidade plena já registrados em São Paulo, Rio de Janeiro, Rio Grande do Sul e Pernambuco.

A aplicação do conceito de vitalidade em um estudo cientométrico sobre a produção do conhecimento científico na área da saúde, no Brasil, revelou que poucas instituições se enquadraram nesta característica. Revelou também que quando há esforço e investimentos, o crescimento e a diversidade logo se apresentaram. Disso, conclui-se com o reconhecimento da necessidade de seguir se investindo na produção científica, através de políticas mais estáveis que possam promover, além da busca por mais igualdade, a própria sobrevivência das instituições, e o desenvolvimento de todo o país.

\section{Referências}

BETTENCOURT, L. M. A. et al. Population modeling of the emergence and development of scientific fields. Scientometrics, Amsterdam, v. 75, n. 3, p. 495-518, 2008.

BÖRNER, K. et al. An introduction to modeling science: basic model types, key definitions, and a general framework for the comparison of process models. In: SCHARNHORST, A.; BORNER, K., et al (Ed.). Models of science dynamics encounters between complexity theory and information sciences. Berlin: Springer, 2012. p.3-22.

BOUCHARD, L. et al. Research on health inequalities: a bibliometric analysis (1966-2014). Social Science \& Medicine, Kidlington, v. 141, p. 100-108, jul. 26, 2015. Disponível em: <http://www.sciencedirect.com/science/article/pii/S0277953615300381>. Acesso em: 14 nov. 2015. 
BOYACK, K. W.; KLAVANS, R. Map of scientific paradigms. In: BÖRNER, K. (Ed.). Atlas of science: visualizing what we know. Cambridge: MIT, 2010. p. $136-37$

BRAZIER, J. et al. Validating the SF-36 health survey questionnaire: new outcome measure for primary care. British Medical Journal, London, v. 305, n. 6846, p. 160-64, 1992.

BUCHELI, V. et al. Growth of scientific production in Colombian universities: an intellectual capital-based approach. Scientometrics, Amsterdam, v. 91, n. 2, p. 369-82, 2012.

CALLON, M.; COURTIAL, J. P.; PENAN, H. Cienciometría: la medición de la actividad científica:de la bibliometría a la vigilancia tecnológica.Gijón: Trea, 1995.

CASTIEL, L. D.; SANZ-VALERO, J. Entre fetichismo e sobrevivência: o artigo científico é uma mercadoria acadêmica? Cadernos de Saúde Pública, Rio de Janeiro, v. 23, n. 12, p. 3041-50, 2007.

COBO, M. J. et al. Science mapping software tools: review, analysis, and cooperative study among tools. Journal of the American Society for Information Science and Technology, Hoboken, v. 62, n. 5, p. 1-21, 2011. Disponível em: <http://dx.doi.org/10.1002/asi.21525>. Acesso em: 14 nov. 2015.

COLE, S. The hierarchy of the sciences? American Journal of Sociology, Chicago, v. 89, n. 1, p. 111-139, 1983.

COLE, S.; COLE, J. R.; SIMON, G. A. Chance and consensus in peer review. Science, Washington, v. 214, n. 4523, p. 881-6, Nov. 20, 1981.

COMMITTEE ON ASSESSING BEHAVIORAL. A strategy for assessing science: behavioral and social research on aging. Washington: National Academies, 2006. Disponível em:

$<$ http://www.nap.edu/openbook.php?record_id=11788>. Acesso em: 14 nov. 2015.

CRUZ, C. H. B. Brazil: reward quality. Nature, London, v. 490, p. 334, 2012.

DANKOSKI, M. E. et al. An expanded model of faculty vitality in academic medicine. Advances in Health Sciences Education, Dordrecht, Dec. 2, 2011.

DING, Y.; CRONIN, B. Popular and/or prestigious? Measures of scholarly esteem. Information Processing \& Management, Oxon, v. 47, n. 1, p. 80-96, 2011. 
FOOTE, M. et al. On the bidirectional relationship between geographic range and taxonomic duration. Paleobiology, Lawrence, v. 34, p. 421-33, 2008.

GLÄNZEL, W.; LETA, J.; THUS, B. Science in Brazil. Part 1: a macro-level comparative study. Scientometrics, Amsterdam, v. 67, n. 1, p. 67-86, 2006.

GREENBERG, S. How citation distortions create unfounded authority: analysis of a citation network. British Medical Journal, London, v. 339, n. b2680, p. 114, 2009.

IRIBARREN-MAESTRO, I. Producción científica y visibilidad de los investigadores de la Universidad Carlos III de Madrid en las Bases de Datos del ISI, 1997-2003. 2006. Tese (Doutorado) - Departamento de Biblioteconomia y Documentación, Universidad Carlos III de Madrid, Getafe, 2006.

JABLONSKI, D. Mass extinctions and macroevolution. Paleobiology, Lawrence, v. 31, n. 192-210, 2005.

KIKUCHI, R. K.; LEAO, Z. M.; OLIVEIRA, M. D. Conservation status and spatial patterns of AGRRA vitality indices in Southwestern Atlantic reefs.

Revista de Biología Tropical, San José, v. 58, Supl. 1, p. 1-31, 2010.

KLAVANS, R.; BOYACK, K. W. Thought leadership: A new indicator for national and institutional comparison. Scientometrics, Amsterdam, v. 75, n. 2, p. 239-250, 2008.

KUHN, T. S. A estrutura das revoluções científicas. 9. ed. São Paulo: Perspectiva, 2005.

LANE, J. Let's make science metrics more scientific. Nature, London, v. 464, p. $488-89,2010$.

LEITE, P.; MUGNAINI, R.; LETA, J. A new indicator for international visibility: exploring Brazilian scientific community. Scientometrics, Amsterdam, v. 88, n. 1, p. 311-319, 2011. Disponível em:

<http://dx.doi.org/10.1007/s11192-011-0379-9>. Acesso em:

LEYDESDORFF, L.; ROTOLO, D.; RAFOLS, I. Bibliometric Perspectives on Medical Innovation using the Medical Subject Headings (MeSH) of PubMed. 2012. Disponível em:〈http://arxiv.org/abs/1203.1006>. Acesso em: abr. 2012.

MAIA, M. F. S. Comunicação científica em ciências da saúde no Brasil: estrutura e dinâmica da produção e indícios de vitalidade. 2014. Tese (Doutorado) - Programa de Pós-Graduação em Comunicação e Informação, Universidade Federal do Rio Grande do Sul, Porto Alegre, 2014. Disponível em: <http://hdl.handle.net/10183/96674>. Acesso em: jul. 2015. 
MALI, F. et al. Dynamic scientific co-authorship networks In:

SCHARNHORST, A.; BORNER, K. et al. (Ed.). Models of science dynamics encounters between complexity theory and information sciences. Berlin: Springer, 2012. p.195-232

MAYR, E. Isto é biologia: a ciência do mundo vivo. São Paulo: Companhia das Letras, 2008.

OLIVEIRA, E. A. et al. Profile and scientific poduction of CNPq researchers in cardiology. Arquivos Brasileiros de Cardiologia, Rio de Janeiro, v. 97, p. 186193, 2011. Disponível em: <http://dx.doi.org/10.1590/S0066782X2011005000086>. Acesso em: 14 nov. 2015.

PARDO, S.; GALVAGNO, M. A.; CERRUTTI, P. Studies of viability and vitality after freezing of the probiotic yeast Saccharomyces boulardii: physiological preconditioning effect. Revista Iberoamericana de Micología, Bilbao, v. 26, n. 2, p. 155-60, jun. 30, 2009.

POBLACIÓN, D. A. et al. (Eds). Revistas científicas: dos processos tradicionais às perspectivas alternativas de comunicação. São Paulo: Ateliê Editorial, 2011.

PORTOCARRERO, V. As ciências da vida: de Canguilhem a Foucault. Rio de Janeiro: Fiocruz, 2009.

RONS, N.; AMEZ, L. Impact vitality: an indicator based on citing publications in search of excellent scientists. Research Evaluation, Oxford, v. 18, n. 3, p. 233-241, 2009.

SANDSTRÖM, U.; SANDSTRÖM, E. The field factor: towards a metric for academic institutions. Research Evaluation, Oxford, v. 18, n. 3, p. 243-250, 2009.

SANTOS, G. C.(Ed.).Fontes de indexação para periódicos científicos: um guia para bibliotecários e editores. Campinas: UNICAMP, 2010.

TENOPIR, C.; KING, D. W. Towards electronic journals: realities for scientists librarians, and publishers. Washington: SLA, 2000.

URBIZAGÁSTEGUI ALVARADO, R. A frente de pesquisa na literatura sobre a produtividade dos autores. Encontros Bibli: revista eletrônica de Biblioteconomia e Ciência da Informação, Florianópolis, v. 14, p. 38-56, 2009.

WAINER, J.; VIEIRA, P. Correlations between bibliometrics and peer evaluation for all disciplines: the evaluation of Brazilian scientists.

Scientometrics, Amsterdam, 2013. 
ZIMAN, J. M. Conhecimento público. Belo Horizonte: Itatiaia, 1979.

\title{
Growth, diversity and survival: the concept of vitality applied to a scientometric study.
}

\begin{abstract}
The paper addresses the structure and dynamics of scientific activities in the Health Sciences in Brazil. It starts with the assumption that vitality in science can be identified by characteristics related to productivity of authors, longevity of institutions, thematic diversity and publication channels. It points out specific features that represent different degrees of vitality in science. Analyzing 117,521 articles by Brazilian authors published between 1987 and 2011 in journals indexed by MEDLINE, vitality characteristics have been identified in each of the 27 states of the country. The results reveal that, in the area of Health Sciences in Brazil, there is much disparity between the states and that the higher levels of scientific vitality are located in the states of São Paulo, Rio de Janeiro and Rio Grande do Sul.
\end{abstract}

Keywords: Scholarly communication. Scholarly communication patterns - Brazil. Health Sciences. Scientometrics.

Recebido em 14/10/2015

Aceito em 21/12/2015 